\title{
Anti-inflammatory effects of CHRNA7 through interacting with adenylyl cyclase 6
}

\author{
Simeng Zhu ${ }^{1}$, Shiqian Huang ${ }^{1}$, Guofang $\mathrm{Xia}^{2}$, Jin $\mathrm{Wu}^{3}$, Yan Shen ${ }^{4}$, Ying Wang ${ }^{1}$, Rennolds \\ Ostrom $^{5}$, Ailian $\mathrm{Du}^{6}$, Chengxing Shen ${ }^{2}$, and Congfeng $\mathrm{Xu}^{1}$ \\ ${ }^{1}$ Shanghai Jiao Tong University School of Medicine \\ ${ }^{2}$ Shanghai Jiaotong University Affiliated Sixth People's Hospital \\ ${ }^{3}$ Shanghai Jiaotong University School of Medicine Xinhua Hospital \\ ${ }^{4}$ Zhengzhou University First Affiliated Hospital \\ ${ }^{5}$ Chapman University \\ ${ }^{6}$ Tongren Hospital Shanghai Jiaotong University School of Medicine
}

July 28, 2020

\begin{abstract}
Background and purpose: Alpha 7 nicotinic acetylcholine receptors (CHRNA7) suppress inflammation through diverse pathways in immune cells, so is potentially involved in a number of inflammatory diseases. However, the detailed mechanisms underlying CHRNA7's anti-inflammatory effects remain elusive. Experimental approach: The anti-inflammatory effects of CHRNA7 agonists in both murine macrophages (RAW 264.7) and bone marrow-derived macrophages (BMDM) stimulated with LPS were examined. The role of adenylyl cyclase 6 (AC6) in Toll-like Receptor 4 (TLR4) degradation was explored via overexpression and knockdown. A mouse model of chronic obstructive pulmonary disease was used to confirm key findings. Key results: Antiinflammatory effects of CHRNA7 were largely dependent on AC6 activation, as knockdown of AC6 considerably abnegated the effects of CHRNA7 agonists while AC6 overexpression promoted them. We found that CHRNA7 and AC6 are co-localized in lipid rafts of macrophages and directly interact. Activation of AC6 led to the promotion of TLR4 degradation. Administration of CHRNA7 agonist PNU282987 attenuated pathological and inflammatory end points in a mouse model of chronic obstructive pulmonary disease (COPD). Conclusion and implications: CHRNA7 inhibit inflammation through activating AC6 and promoting degradation of TLR4. The use of CHRNA7 agonists might represent a novel therapeutic approach for treating COPD and likely other inflammatory diseases.
\end{abstract}

\section{Hosted file}

Manuscript - AChR \& AC6 20200719.pdf available at https://authorea.com/users/346688/articles/ 472613-anti-inflammatory-effects-of-chrna7-through-interacting-with-adenylyl-cyclase-6 

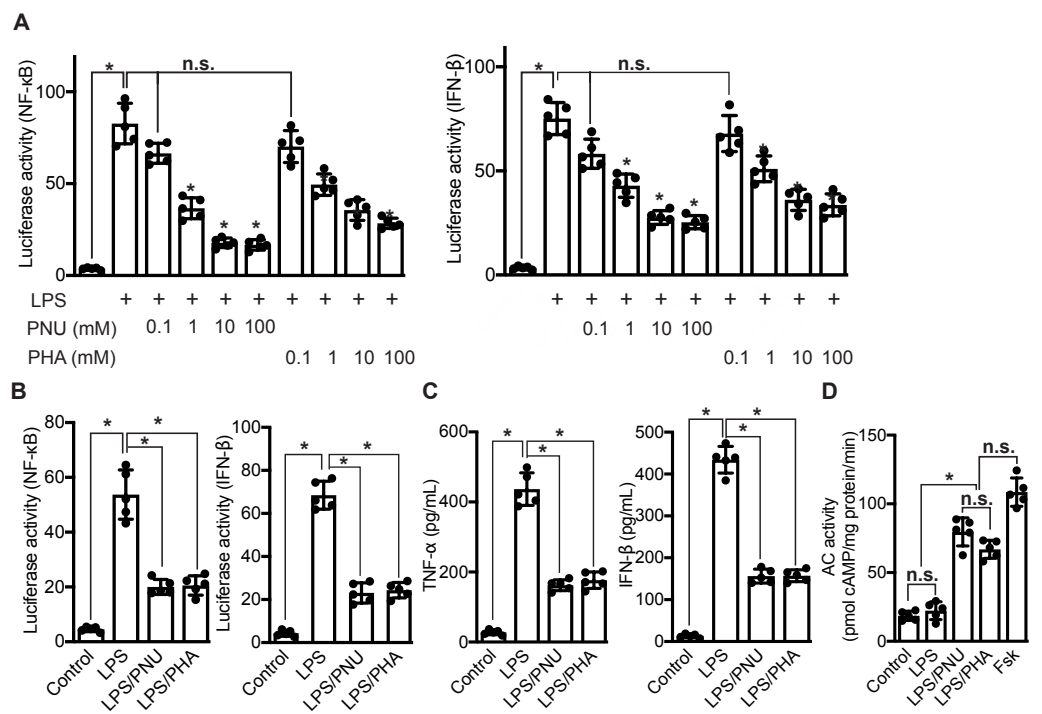